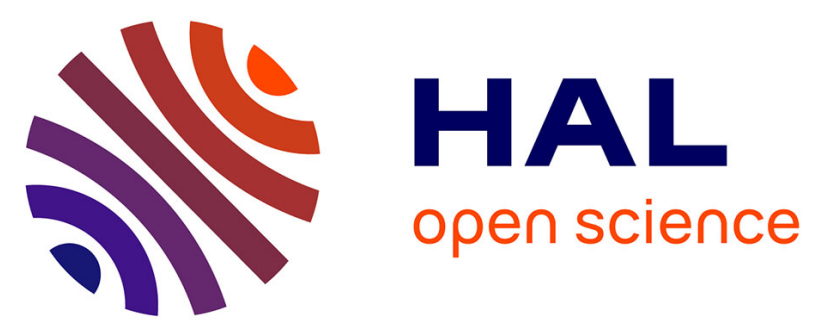

\title{
Nanoscale aluminum plasmonic waveguide with monolithically integrated germanium detector
}

M. Sistani, M. Bartmann, N. Güsken, R. Oulton, H. Keshmiri, M. Seifner, S. Barth, N. Fukata, M. Luong, M. den Hertog, et al.

\section{- To cite this version:}

M. Sistani, M. Bartmann, N. Güsken, R. Oulton, H. Keshmiri, et al.. Nanoscale aluminum plasmonic waveguide with monolithically integrated germanium detector. Applied Physics Letters, 2019, 115 (16), pp.161107. 10.1063/1.5115342 . hal-03003402

\section{HAL Id: hal-03003402 https://hal.science/hal-03003402}

Submitted on 19 Nov 2020

HAL is a multi-disciplinary open access archive for the deposit and dissemination of scientific research documents, whether they are published or not. The documents may come from teaching and research institutions in France or abroad, or from public or private research centers.
L'archive ouverte pluridisciplinaire HAL, est destinée au dépôt et à la diffusion de documents scientifiques de niveau recherche, publiés ou non, émanant des établissements d'enseignement et de recherche français ou étrangers, des laboratoires publics ou privés. 


\section{Nanoscale aluminum plasmonic waveguide with monolithically integrated germanium detector}

Cite as: Appl. Phys. Lett. 115, 161107 (2019); https://doi.org/10.1063/1.5115342

Submitted: 17 June 2019 . Accepted: 29 September 2019 . Published Online: 15 October 2019

M. Sistani (D), M. G. Bartmann (D), N. A. Güsken (D), R. F. Oulton (D), H. Keshmiri (D), M. S. Seifner (D), S. Barth (D), N. Fukata (D), M. A. Luong (D), M. I. den Hertog (D), and A. Lugstein (D)
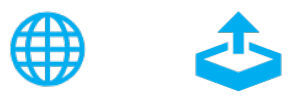

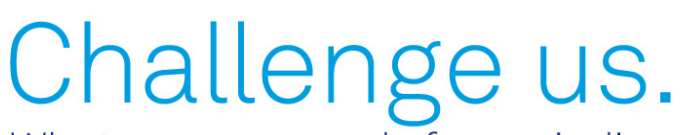

What are your needs for periodic signal detection?

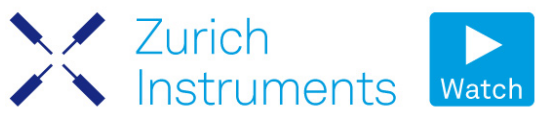

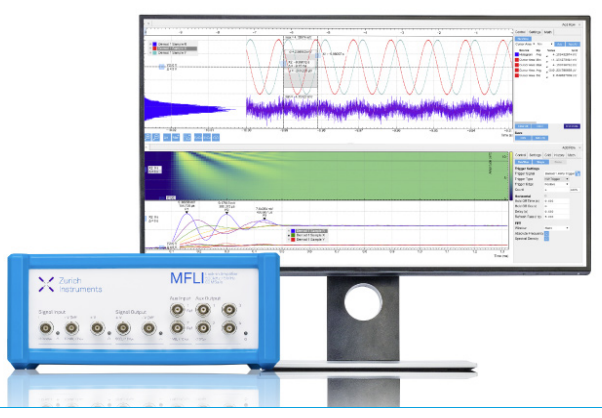

Appl. Phys. Lett. 115, 161107 (2019); https://doi.org/10.1063/1.5115342

115, 161107 


\title{
Nanoscale aluminum plasmonic waveguide with monolithically integrated germanium detector
}

\author{
Cite as: Appl. Phys. Lett. 115, 161107 (2019); doi: 10.1063/1.5115342 \\ Submitted: 17 June 2019 - Accepted: 29 September 2019 . \\ Published Online: 15 October 2019
}

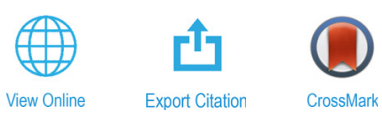

M. Sistani, ${ }^{1, a)}$ (D) M. G. Bartmann,, ${ }^{1, a)}$ (D) N. A. Güsken, ${ }^{2}$ (iD R. F. Oulton, ${ }^{2}$ (D) H. Keshmiri, ${ }^{1}$ (D) M. S. Seifner, ${ }^{3}$

S. Barth, ${ }^{4}$ (D) N. Fukata, ${ }^{5}$ (D) M. A. Luong, ${ }^{6}$ (D) M. I. den Hertog, ${ }^{7}$ (iD) and A. Lugstein ${ }^{1, b)}$ (iD)

\author{
AFFILIATIONS \\ IIInstitute of Solid State Electronics, Technische Universität Wien, Gußhausstraße 25-25a, 1040 Vienna, Austria \\ ${ }^{2}$ The Blackett Laboratory, Department of Physics, Imperial College London, London SW7 2AZ, United Kingdom \\ ${ }^{3}$ Institute of Materials Chemistry, Technische Universität Wien, Getreidemarkt 9, 1060 Vienna, Austria \\ ${ }^{4}$ Physikalisches Institut, Goethe University Frankfurt, Max-von-Laue-Str. 1, 60438 Frankfurt am Main, Germany \\ ${ }^{5}$ International Center for Materials Nanoarchitectonics, National Institute for Materials Science, 1-1 Namiki, 305-0044 Tsukuba, \\ Japan \\ ${ }^{6}$ University Grenoble Alpes, CEA, INAC, MEM, F-38000 Grenoble, France \\ ${ }^{7}$ Univ. Grenoble Alpes, CNRS, Grenoble INP, Institut Néel, 38000 Grenoble, France \\ ${ }^{a)}$ Contributions: M. Sistani and M. G. Bartmann contributed equally to this work. \\ ${ }^{\text {b) }}$ Author to whom correspondence should be addressed: alois.lugstein@tuwien.ac.at
}

\begin{abstract}
Surface plasmon polaritons have rapidly established themselves as a promising concept for molecular sensing, near-field nanoimaging, and transmission lines for emerging integrated ultracompact photonic circuits. In this letter, we demonstrate a highly compact surface plasmon polariton detector based on an axial metal-semiconductor-metal nanowire heterostructure device. Here, an in-coupled surface plasmon polariton propagates along an aluminum nanowire waveguide joined to a high index germanium segment, which effectively acts as a photoconductor at low bias. Based on this system, we experimentally verify surface plasmon propagation along monocrystalline Al nanowires as thin as $40 \mathrm{~nm}$ in diameters. Furthermore, the monolithic integration of plasmon generation, guiding, and detection enables us to examine the bending losses of kinked waveguides. These systematic investigations of ultrathin monocrystalline Al nanowires represent a general platform for the evaluation of nanoscale metal based waveguides for transmission lines of next generation high-speed ultracompact on-chip photonic circuits.
\end{abstract}

Published under license by AIP Publishing. https://doi.org/10.1063/1.5115342

The ever-increasing demand for more-compact and thus costeffective chemical sensing solutions anticipates a roadmap leading to a monolithic integration of optical components and functionalities into ultracompact integrated photonic circuits. ${ }^{1-7}$ Consequently, addressing demands with respect to operation speeds, efficiencies, and critical feature sizes rivaling electronics has triggered intense research interest in photonic components scaled beyond the diffraction limit of light..$^{5}$ In this context, surface plasmon polaritons (SPPs), collective oscillations of electrons at metal-dielectric interfaces, constitute a promising concept, capable of concentrating and routing optical signals using subwavelength structures. ${ }^{8,9}$ Networks of kinked and branched nanowires (NWs) enable redirection of SPPs within a photonic circuit and thus support compact interferometric routing and logic operations. ${ }^{10-12}$ With respect to the waveguide material, unlike commonly used metals for studying plasmonic effects such as $\mathrm{Ag}$ and $\mathrm{Au}, \mathrm{Al}$ features unique material properties, enabling strong plasmon resonances ranging from the visible into ultraviolet. ${ }^{1,14}$ Thus, its natural abundance, chemical stability, low cost, and amenability to manufacturing processes and CMOS compatibility make $\mathrm{Al}$ a promising candidate for scalable plasmonic applications. ${ }^{13,15}$ However, propagation losses, radiation from leaky plasmon modes, and scattering by surface roughness are inevitable in realistic nanostructures. ${ }^{16}$ Hence, the geometry, structural size, and crystallinity of a NW waveguide are of crucial importance for plasmon propagation lengths. ${ }^{17}$ Direct imaging of SPP propagation along NWs is commonly accomplished by scanning near-field optical microscopy, ${ }^{17,18}$ limited to the spatial resolution of this optical method. ${ }^{10}$ Thus, in order to investigate the expected short propagation length of SPPs in ultrathin metallic NWs, ${ }^{10,19}$ we explored a near-field plasmon electrical detection 
scheme, ${ }^{20}$ based on an axial metal-semiconductor-metal (M-S-M) NW heterostructure device architecture.

The $\mathrm{Al}-\mathrm{Ge}-\mathrm{Al} \mathrm{NW}$ heterostructures were synthetized on $40 \mathrm{~nm}$ thick $\mathrm{Si}_{3} \mathrm{~N}_{4}$ TEM-membranes ${ }^{21}$ by a thermally induced exchange reaction of single-crystalline Ge NWs covered by a passivating $\mathrm{Al}_{2} \mathrm{O}_{3}$ shell and lithographically defined $\mathrm{Al}$ contact pads [see Fig. 1(a) and the SEM image in the inset]. The NW growth and heterostructure device formation mechanism is discussed in the supplementary material and in detail in the former works of Kral et al. ${ }^{22}$ and El Hajraoui et al. ${ }^{23}$ The single crystalline Al NW plasmonic waveguide and the $\mathrm{Ge}$ NW segment acting as an electronic plasmon detector are connected via an abrupt metal-semiconductor interface [see Fig. 1(b) and the supplementary material]. An efficient launching of SPPs along the Al NW is difficult to achieve due to the inherent size mismatch between the photonic and plasmonic modes. Here, plasmon excitation by phase-matching ${ }^{24,25}$ of the $\lambda=532 \mathrm{~nm}$ laser light to SPPs was achieved by an on-chip design of a focused diffraction grating coupler (FGC) $)^{26,27}$ patterned in the $\mathrm{Si}_{3} \mathrm{~N}_{4}$-TEM-membrane atop of the lithographically defined $\mathrm{Al}$ contacts using focused ion beam nanomachining [see Fig. 1(c)]. Unlike regular gratings supporting flat wave-fronts, an FGC generates converging wave-fronts, enabling improved coupling into the narrow NW waveguide. ${ }^{28}$ The overall working principle is as follows: SPPs generated by the FGC patterned atop of the Al contact pads are guided along the $\mathrm{c}-\mathrm{Al} \mathrm{NW}$ plasmon waveguide acting also as an electrical contact of the Ge sensing element. At the Al-Ge interface, SPPs decay and generate excited carriers ${ }^{29}$ in the adjacent $\mathrm{Ge}$ segment. $^{20}$ By applying a bias voltage $\left(\mathrm{V}_{\text {Bias }}\right)$ as shown in Fig. 1(a), these charge carriers induce a current proportional to the SPP intensity, enabling the detection of the plasmon signals by direct electric means. The length of the $\mathrm{c}$ - $\mathrm{Al}$ waveguide $\left(\mathrm{L}_{\mathrm{c}-\mathrm{Al}}\right)$ as well as the $\mathrm{Ge}$ segment $\left(\mathrm{L}_{\mathrm{Ge}}\right)$ can be tuned by the contact gap $(\mathrm{L})$ [see the SEM image in the inset of Fig. 1(a)] and the processing parameters, i.e., duration and temperature of the thermally induced exchange reaction. As

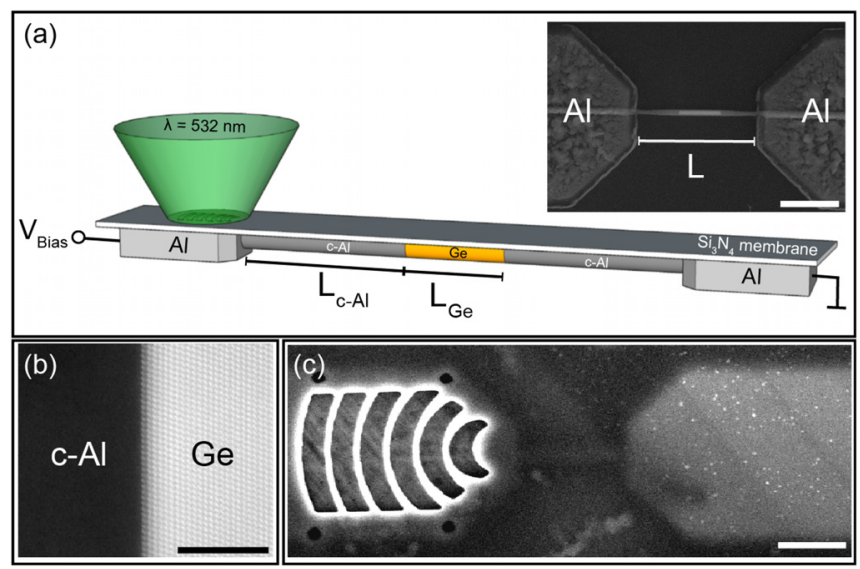

FIG. 1. (a) Schematic illustration of the Al-Ge-Al NW heterostructure device architecture fabricated on a $40 \mathrm{~nm}$ thick $\mathrm{Si}_{3} \mathrm{~N}_{4}$-membrane. The SEM image (the scale bar is $1 \mu \mathrm{m}$ ) in the inset shows the Al-Ge-Al NW heterostructure connected to macroscopic Al pads with a gap of about $\mathrm{L}=2 \mu \mathrm{m}$. (b) HRTEM image showing the atomically sharp and defect free Al-Ge heterojunction. The scale bar is $5 \mathrm{~nm}$. (c) SEM image showing the FGC patterned in the $\mathrm{Si}_{3} \mathrm{~N}_{4}$-membrane atop of the lithographically defined Al contacts. The scale bar is $1 \mu \mathrm{m}$. shown in the supplementary material, the length of the c-Al section of a NW can be adjusted by successive annealing steps, enabling the investigation of SPP propagation for one and the same device as a function of the $\mathrm{c}-\mathrm{Al}$ waveguide length.

To verify the working principle and the spectral responsivity of the device, a tunable laser was focused on the FGC while simultaneously measuring the current through the biased device. The plot in Fig. 2 shows the normalized current induced in the Ge segment by plasmons generated by excitation of the FGC with a laser wavelength between $\lambda=480 \mathrm{~nm}$ and $1100 \mathrm{~nm}$ (see the inset of Fig. 2). As the FGC was optimized for green laser light, a distinct maximum can be observed at about $\lambda=532 \mathrm{~nm}$, as well as the second order peak around $\lambda=1064 \mathrm{~nm}$.

To clarify the local origin of the plasmon induced current, the laser was scanned across the heterostructure device as indicated in the top panel of Fig. 3(a). The lower panel shows the corresponding current for the scanning laser being polarized perpendicular with respect to the NW axis. A distinct current peak further denoted $I_{\text {plas }}$ appears when the laser matches the position of the FGC. SPPs generated at the FGC and effectively coupled into the c-Al NW generate excited carriers in the adjacent Ge segment, resulting in a bias dependent current.

Finite difference time domain (FDTD) simulations revealed that incident light with a polarization perpendicular to the grating couples best to the fundamental mode of the NW-membrane system, which is predominately TM polarized, i.e., in the y-direction [see mode electric field distributions in Fig. 3(b)]. The different plasmon mode contributions in the respective field polarization $(x, y, z)$ are depicted in Fig. 3(b). The second peak appears when the laser beam directly illuminates the electrically biased Ge segment forming electron-hole pairs, resulting in a common photocurrent, denoted $\mathrm{I}_{\mathrm{pc}}$. Since there is no FGC incorporated at the opposite $\mathrm{Al}$ contact, the signal at this position equals the dark current.

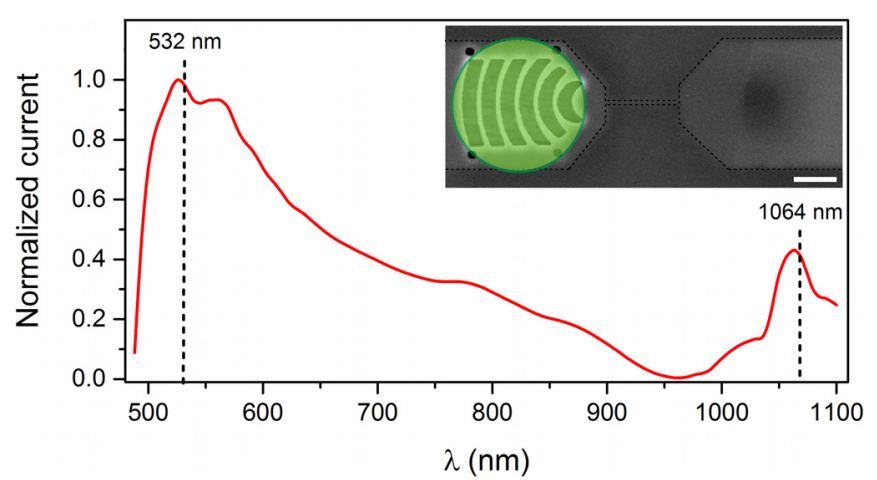

FIG. 2. Spectral responsivity of the FGC excited by a tunable laser, operating in the wavelength range between $\lambda=480 \mathrm{~nm}$ and $1100 \mathrm{~nm}$, while simultaneously biasing the device with $\mathrm{V}_{\mathrm{Bias}}=100 \mathrm{mV}$. The diameter and length of the c-Al NW waveguide attached to the FGC were $40 \mathrm{~nm}$ and $700 \mathrm{~nm}$, respectively. The measurement was conducted at room temperature and ambient conditions, and the data were corrected with respect to the wavelength dependent laser output power. The SEM image in the inset shows the overall contact arrangement of the Al-Ge-Al NW heterostructure device. The scale bar is $1 \mu \mathrm{m}$. The green spot indicates the laser position for exciting the FGC by the tunable laser. 
(a)

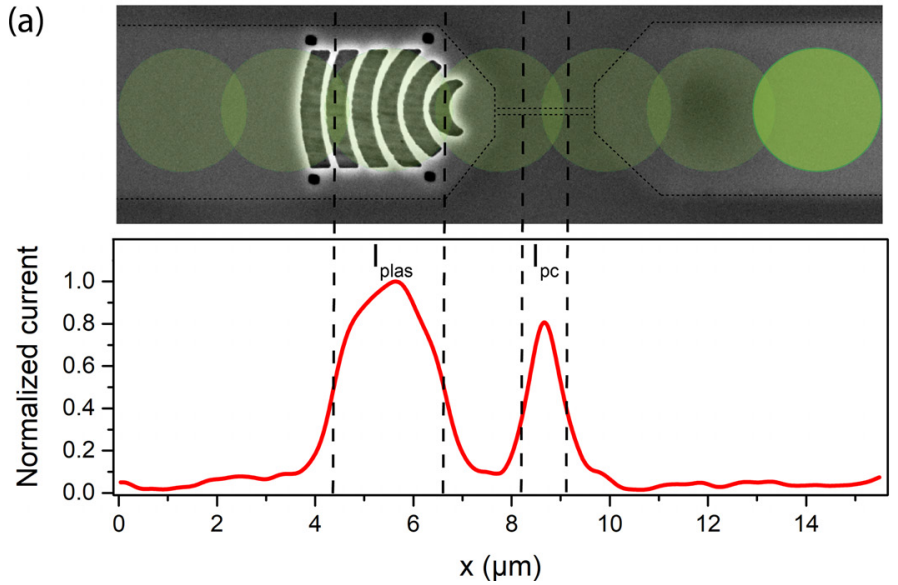

(b)
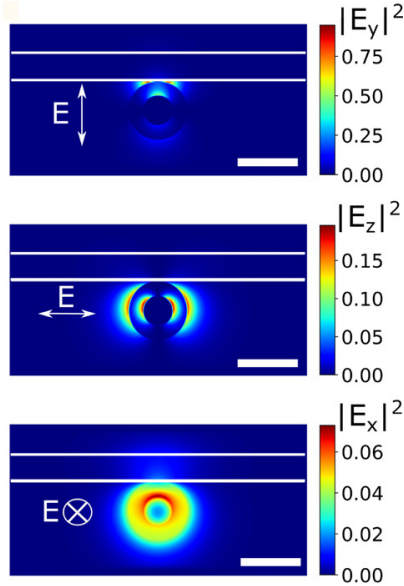

FIG. 3. (a) The upper panel shows an SEM image of the contact arrangement of the Al-Ge-Al NW heterostructure device with the FGC patterned atop of the left lithographically defined Al contact with superimposed grating and beam contours. The lower panel shows the respective line scan across the device starting from the FGC contact toward the opposite contact measuring both the plasmon induced current and photocurrent for TM polarized laser excitation $\left(V_{\text {Bias }}=100 \mathrm{mV}\right)$. (b) FDTD simulations of the electric field intensity for the $\left|E_{y}\right|^{2},\left|E_{z}\right|^{2}$, and $\left|E_{x}\right|^{2}$ field distributions of the fundamental mode in the NW-Si $\mathrm{N}_{4}$ membrane system. The scale bar is $60 \mathrm{~nm}$; the white arrows indicate the predominant field polarization, and the white lines are the contours of the membrane.

The FGC plasmon generator in combination with the extremely sensitive near-field electrical Al-Ge-Al plasmon detector device allows the propagation length of SPPs in ultrathin c-Al NW waveguides to be measured with a high spatial resolution. Figure 4(a) shows the plasmon induced currents for devices with various lengths of the c-Al SPP waveguides $\left(\mathrm{L}_{\mathrm{c}-\mathrm{Al}}\right)$ between $450 \mathrm{~nm}$ and $1085 \mathrm{~nm}$ [see the inset of Fig. 4(a)]. Assuming that $\mathrm{I}_{\text {plas }}$ is directly correlated with the SPP intensity, a decrease in more than two orders of magnitude for an only $600 \mathrm{~nm}$ long waveguide indicates extreme damping for such ultrathin $\mathrm{Al}$ NWs. The exponential fit of the experimental data reveals a propagation length of $\mathrm{L}_{\mathrm{SPP}}=140 \mathrm{~nm}$. Based on FDTD simulations, an imaginary part of the effective refractive index of the fundamental mode in the $\mathrm{c}$ - $\mathrm{Al} \mathrm{NW}$ of $\mathfrak{I}\left(\mathrm{n}_{\text {eff. }}\right) \approx 0.3$ at $\lambda=532 \mathrm{~nm}$ was extracted from which a propagation length of $\mathrm{L}_{\mathrm{SPP}, \text { calc. }}=\frac{\lambda}{4 \pi \mathfrak{I}\left(\mathrm{n}_{\mathrm{eff} .}\right)}=141 \mathrm{~nm}$
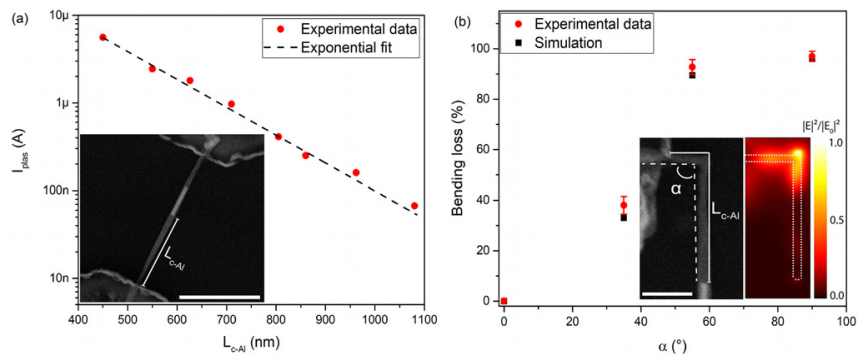

FIG. 4. (a) Plasmon induced current as a function of the length $\mathrm{L}_{c-A l}$ of the $c-A l$ waveguide. The inset shows an SEM image of a heterostructure device with an indicated length of the C-Al waveguide of $\mathrm{L}_{\mathrm{C}-\mathrm{Al}}=950 \mathrm{~nm}$. The scale bar is $1 \mu \mathrm{m}$. (b) Experimentally determined and calculated bending losses depending on the kinking angle $\alpha$ for the M-S-M devices with an overall c-Al waveguide length of $\mathrm{L}_{c-\mathrm{Al}}$ $\approx 700 \mathrm{~nm}$. The left inset shows the SEM image of a device with a $90^{\circ}$ kinked $\mathrm{C}-\mathrm{Al}$ plasmon waveguide. The scale bar is $200 \mathrm{~nm}$. The right inset shows the respective FDTD simulation plot. All measurements were conducted for $V_{\text {Bias }}=100 \mathrm{mV}$, $\mathrm{E}_{\mathrm{L}}=340 \mu \mathrm{W} / \mu \mathrm{m}^{2}$, and $\lambda=532 \mathrm{~nm}$ at room temperature and ambient conditions. could be calculated. In the simulation, an ultrathin Ge shell originating from the fabrication process and surrounding the $\mathrm{Al} \mathrm{NW}$ was also taken into account, which is consistent with the TEM analysis provided by El Hajraoui et al. ${ }^{23}$

Thus, although the investigated $\mathrm{Al} \mathrm{NWs}$ are monocrystalline and rodlike with a smooth surface, ${ }^{30}$ the confinement of SPPs in such ultrathin NWs results in high propagation loss, ${ }^{16}$ limiting the transmission length for on-chip communication even for straight NWs. However, simulations show that a manifold enhancement of the SPP propagation length can be achieved by tuning the NW diameter. ${ }^{31}$ For our system, an increased $\mathrm{Al} \mathrm{NW}$ diameter of $100 \mathrm{~nm}$ would lead to a propagation length of $470 \mathrm{~nm}$. Simulations showing the dependency of the diameter of the c-Al NW waveguides on the SPP propagation length are provided in the supplementary material. Future high-speed ultracompact photonic components will require waveguide bends capable of redirecting signals within a circuit with minimum footprint and as little losses as possible. ${ }^{10,16}$ In order to investigate such broken symmetry waveguides, ${ }^{10}$ kinked c-Al SPP waveguides with an overall length of $\mathrm{L}_{\mathrm{c}-\mathrm{Al}} \approx 700 \mathrm{~nm}$ exhibiting approximately zero radius of curvature and inclination angles between $\alpha=0^{\circ}$ and $90^{\circ}$ were integrated in the M-S-M devices. The inset in Fig. 4(b) shows an SEM image and the FDTD simulation plot of an exemplarily device with a $90^{\circ}$ inclined c-Al plasmon waveguide. Additional SEM-images showing heterostructure devices with $35^{\circ}$ and $55^{\circ}$ inclined c-Al plasmon waveguides are displayed in the supplementary material. The disturbance of SPP propagation at the kink is clearly observable in the simulation plot. Propagating SPPs are effectively scattered into radiating photons at the kink, resulting in a drop of the transmitted power. ${ }^{16,32,33}$ By calculating the electric fields at the end facet of the $700 \mathrm{~nm}$ long c-Al NW, the resultant bending losses were determined to be $33 \%, 89 \%$, and $96 \%$ for kink angles of $\alpha=35^{\circ}, 55^{\circ}$, and $90^{\circ}$, respectively.

Experimentally, due to the enhanced scattering at the kinks, less plasmons pass the $700 \mathrm{~nm}$ long c-Al NW on their way to the Al-Ge interface. The plasmon induced current thus decreases with increasing kinking angles. The measured bending losses shown in the main plot 
of Fig. 4(b) are in reasonable agreement with the simulated values. The shown data points correspond to the mean values over three devices for each of the investigated kink-angles. This clearly demonstrates that bending losses in ultrathin metal waveguides are a crucial factor for the design of transmission lines within ultracompact photonic circuits.

In conclusion, we have demonstrated the effective coupling and guiding of light over ultrathin c-Al NWs. To measure the plasmonic propagation characteristics, we explored an extremely sensitive near-field electrical plasmon detector based on an axial Al-Ge-Al NW heterostructure. Plasmon excitation by phase-matching light to SPPs was achieved by FGCs tuned for an excitation wavelength of $\lambda$ $=532 \mathrm{~nm}$. Based on this system, we experimentally determined a propagation length of $\mathrm{L}_{\mathrm{SPP}}=140 \mathrm{~nm}$ for the confined SPPs in monocrystalline $40 \mathrm{~nm}$ thin $\mathrm{Al}$ NWs. Most notably, with respect to waveguide bends required for on-chip routing of signals within photonic networks, we assessed the bending losses for kinked c-Al NWs. Thus, our monolithic approach is an important step toward highly efficient plasmon sensing applications that eliminate the need for far-field detection.

See the supplementary material for Ge NW synthesis, device fabrication, and electrical and optical device characterization; detailed description of a HRTEM image recorded at the Al-Ge interface; discussion of the approach to control the length of the c-Al NWs and the respective change of the plasmon induced currents by successive thermal annealing; images of devices with $35^{\circ}, 55^{\circ}$, and $90^{\circ}$ inclined c-Al plasmon waveguides; and simulations showing the dependency of the diameter of the $\mathrm{c}-\mathrm{Al} \mathrm{NW}$ waveguides on the SPP propagation length.

The authors gratefully acknowledge financial support from the Austrian Science Fund (FWF): Project No: P28175. The authors further thank the Center for Micro- and Nanostructures, TU Wien, for providing the cleanroom facilities. S.B. thanks the Deutsche Forschungsgemeinschaft (DFG, German Research Foundation) for funding Project No. 413940754. We acknowledge support from the Laboratoire d'excellence LANEF in Grenoble (No. ANR-10-LABX51-01). We benefitted from the access to the Nano characterization platform (PFNC) in CEA Minatec Grenoble and Nanofab from Institut NEEL, Grenoble. We acknowledge funding from the ANR in the T-ERC project e-See. We acknowledge the Leverhulme Trust (No. RPG-2016-064) and the Engineering and Physical Sciences Research Council (EPSRC) as well as the Reactive Plasmonic Program Grant (No. EP/M013812/353 1).

\section{REFERENCES}

${ }^{1}$ B. Schwarz, P. Reininger, D. Ristanić, H. Detz, A. M. Andrews, W. Schrenk, and G. Strasser, Nat. Commun. 5, 4085 (2014).

${ }^{2}$ K. Lodewijks, J. Ryken, W. Van Roy, G. Borghs, L. Lagae, and P. Van Dorpe, Plasmonics 8, 1379 (2013)
${ }^{3}$ R. F. Oulton, V. J. Sorger, D. A. Genov, D. F. P. Pile, and X. Zhang, Nat. Photonics 2, 496 (2008).

${ }^{4}$ S. Kawata, Y. Inouye, and P. Verma, Nat. Photonics 3, 388 (2009).

${ }^{5}$ V. J. Sorger, R. F. Oulton, R.-M. Ma, and X. Zhang, MRS Bull. 37, 728 (2012).

${ }^{6}$ W. L. Barnes, A. Dereux, and T. W. Ebbesen, Nature 424, 824 (2003).

${ }^{7}$ J. Appenzeller, J. Knoch, M. T. Bjork, H. Riel, H. Schmid, and W. Riess, IEEE Trans. Electron Devices 55, 2827 (2008).

${ }^{8}$ E. Ozbay, Science 311, 189 (2006).

${ }^{9}$ H. A. Atwater and A. Polman, Nat. Mater. 9, 205 (2010).

${ }^{10}$ D. Pan, H. Wei, Z. Jia, and H. Xu, Sci. Rep. 4, 4993 (2015).

${ }^{11}$ H. Wei, Z. Li, X. Tian, Z. Wang, F. Cong, N. Liu, S. Zhang, P. Nordlander, N. J. Halas, and $\mathrm{H}$. Xu, Nano Lett. 11, 471 (2011).

${ }^{12}$ H. Wei, Z. Wang, X. Tian, M. Käll, and H. Xu, Nat. Commun. 2, 387 (2011).

${ }^{13}$ M. W. Knight, N. S. King, L. Liu, H. O. Everitt, P. Nordlander, and N. J. Halas, ACS Nano 8, 834 (2014).

${ }^{14}$ D. Gérard and S. K. Gray, J. Phys. D 48, 184001 (2015).

${ }^{15}$ C. Langhammer, M. Schwind, B. Kasemo, and I. Zorić, Nano Lett. 8, 1461 (2008).

${ }^{16}$ H. Wei, D. Pan, S. Zhang, Z. Li, Q. Li, N. Liu, W. Wang, and H. Xu, Chem. Rev. 118, 2882 (2018).

${ }^{17}$ H. Ditlbacher, A. Hohenau, D. Wagner, U. Kreibig, M. Rogers, F. Hofer, F. R. Aussenegg, and J. R. Krenn, Phys. Rev. Lett. 95, 257403 (2005).

${ }^{18}$ D. W. Pohl, W. Denk, and M. Lanz, Appl. Phys. Lett. 44, 651 (1984).

${ }^{19}$ A. Paul, D. Solis, K. Bao, W.-S. Chang, S. Nauert, L. Vidgerman, E. R. Zubarev, P. Nordlander, and S. Link, ACS Nano 6, 8105 (2012).

${ }^{20}$ A. L. Falk, F. H. L. Koppens, C. L. Yu, K. Kang, N. de Leon Snapp, A. V. Akimov, M.-H. Jo, M. D. Lukin, and H. Park, Nat. Phys. 5, 475 (2009).

${ }^{21}$ M. den Hertog, F. Donatini, R. McLeod, E. Monroy, C. Sartel, V. Sallet, and J. Pernot, Nanotechnology 29, 025710 (2018).

${ }^{22}$ S. Kral, C. Zeiner, M. Stöger-Pollach, E. Bertagnolli, M. I. den Hertog, M. Lopez-Haro, E. Robin, K. El Hajraoui, and A. Lugstein, Nano Lett. 15, 4783 (2015).

${ }^{23}$ K. El Hajraoui, M. A. Luong, E. Robin, F. Brunbauer, C. Zeiner, A. Lugstein, P. Gentile, J.-L. Rouvière, and M. Den Hertog, Nano Lett. 19, 2897 (2019).

${ }^{24}$ S. A. Maier, Plasmonics: Fundamentals and Applications (Springer Science \& Business Media, 2007).

${ }^{25}$ S. T. Koev, A. Agrawal, H. J. Lezec, and V. A. Aksyuk, Plasmonics 7, 269 (2012).

${ }^{26}$ H. Keshmiri, "Loss mitigation in binary-sequenced plasmonic waveguides," Ph.D. thesis (Institute of Solid State Electronics, TU Wien, 2017).

${ }^{27}$ F. Van Laere, W. Bogaerts, D. Taillaert, P. Dumon, D. Van Thourhout, and R. Baets, in OFC/NFOEC 2007-2007 Conference on Optical Fiber Communication and the National Fiber Optics Engineering Conference (IEEE, 2007), pp. 1-3.

${ }^{28}$ N. A. Güsken, M. P. Nielsen, N. B. Nguyen, S. A. Maier, and R. F. Oulton, Opt. Express 26, 30634 (2018).

${ }^{29}$ B. Y. Zheng, H. Zhao, A. Manjavacas, M. McClain, P. Nordlander, and N. J. Halas, Nat. Commun. 6, 7797 (2015).

${ }^{30}$ F. M. Brunbauer, E. Bertagnolli, J. Majer, and A. Lugstein, Nanotechnology 27, 385704 (2016).

${ }^{31}$ J. Takahara, S. Yamagishi, H. Taki, A. Morimoto, and T. Kobayashi, Opt. Lett. 22, 475 (1997).

${ }^{32}$ A. W. Sanders, D. A. Routenberg, B. J. Wiley, Y. Xia, E. R. Dufresne, and M. A. Reed, Nano Lett. 6, 1822 (2006).

${ }^{33}$ Y. Ding, X. Song, P. Jiang, R. Jiao, L. Wang, L. Yu, and J. Zhang, Laser Photonics Rev. 12, 1700073 (2018). 